Journal of Physics and Its Applications

Journal homepage: https://ejournal2.undip.ac.id/index.php/ipa/index

\title{
Evaluation and Implementation of Otsu and Active Contour Segmentation in Contrast- Enhanced Cardiac CT Images
}

\author{
Arvi Razanata ${ }^{*}$, Prawito Prajitno², Djarwani Soeharso Soejoko² \\ ${ }^{1}$ Graduate Physics Study Program, Faculty of Mathematics and Natural Sciences, University of Indonesia, Depok, Indonesia \\ ${ }^{2}$ Department of Physics, Faculty of Mathematics and Natural Sciences, University of Indonesia, Depok, West Java, Indonesia \\ ${ }^{*}$ Corresponding author:arvi.razanata@sci.ui.ac.id
}

\section{A R T I C L E I N F O}

\section{Article history:}

Received: 29 March 2021

Accepted: 30 April 2021

Available online: 28 May 2021

\section{Keywords:}

Segmentation

Volume Quantification

CT Cardiac

Active Contour

\begin{abstract}
A B S T R A C T
The CT cardiac acquisition process is usually conducted by using an additional image with contrast medium that is injected inside the body and reconstructed by a radiologist using an integrated CT Scan software with the aim to find the morphology and volume dimension of the heart and coronary arteries. In fact, the data obtained from the hospital are raw data without segmented contour from a radiologist. For the purpose of automation, dataset is needed to be used as input data for further program development. This study is focused on the evaluation of the segmentation results of CT cardiac images using Otsu threshold and active contour algorithm with the aim to make a dataset for the heart volume quantification that can be used interactively as an alternative to integrated CT scan software. 2D contrast enhanced cardiac CT from 6 patients using image processing techniques was run on Matlab software. Of the 689 slices that was used, as many as (73.75 \pm 19.41)\% of CT cardiac slices have been segmented properly, (19.15 \pm $19.61) \%$ of the slices that were segmented included the spine bone, $(1.36 \pm$ $0.98) \%$ of the slices did not include all region of the heart, $(16.58 \pm 15.26) \%$ of the slices included other organs with the consistency from the measurement proven from inter-observer variability to produce $r=0,9941$. The result is due to the geometry influence from the diameter of the patient's body thickness that tends to be thin.
\end{abstract}

\section{Introduction}

One of the causes of death of population globally is cardiac disease [1]. According to the statistics in 2016, Indonesia with an estimate population of 261 million people, more than 470.000 people die due to coronary artery disease every year[2].Imaging modality such as CT scan is used as an early detection for coronary artery disease. The calcium score is used to evaluate the risk of coronary artery disease[3, 4]. The image acquisition of CT scanproducesnon-contrast cardiac CT and contrast cardiac CT images. Non-contrast images are used to detect calcium from coronary arteries and contrast enhanced images are used for the morphology of the heart and coronary arteries [5].

The heart is a hollow, fibromuscular organ in the shape of an irregular cone located in the middle mediastinum, with its long axis lying obliquely along a line running from the left mid-clavicular line anteriorly, to the right mid-scapular line posteriorly. A third of the heart lies to the right of median(sagittal) plane of the body and two-thirds to the left of this plane [6]. The image reconstruction process to obtain the morphology and volume dimension of the heart is conducted by a radiologist using an integrated CT scan software. In fact, not everyone have access to image processing software in the hospital for a long period of time. Therefore automatic image processing method is needed for quantification of heart volume that is computer based, require many data, and data slices that are not readily available in hospitals to be used as dataset or data training for automation on Computer Aided Diagnosis (CAD). Studies regarding image segmentation of CT cardiac images have been published[7-10]. Nevertheless, many published methods have disadvantages for routine clinical practice such as require additional data images to be acquired[8].

Image segmentation plays a fundamental and important role in image analysis and computer vision. Its purpose is to divide a given image into several parts where each part is homogeneous as the aspect of certain characteristics(i.e., intensity, color, and texture)[11, 12]. The basic approach of segmentation is to select an appropriate threshold value from a gray scale image, we use Otsu thresholding algorithm to segment the heart because that method is the simplest and one of the most widely used techniques for image segmentationwith 
the main purpose is to simplify or change the respective image sample into an easily analyzed image [13].Several approaches based on deformable models were dedicated to the image segmentation in CT images i.e., Chan-Vese, $L B F$, Active Contour.As a result, active contour can cover the entire area of a given object compared to LBF and Chan-Vese[14]. According to the nature of constrains, active contour models can be roughly categorized into the edgebased models and region based models[15]. Active contour may be used to detect the edges forming an object boundary, given an initial guess (i.e., initial contour)[16].

Most cardiac segmentation techniques treat 2D segmentation and 3D multiplanar reconstruction as two separate processes $[17,18]$. These processes achieve volumetric reconstruction by first applying a 2D segmentation approach independently for each slice, and then reconstructing these 2D segmented image stacks into 3D objects [19]. Based on that, we propose and evaluate a combination of Otsu threshold and active contour algorithm of the cardiac images from CT scans to make a dataset which can be used for automatic heart volume quantification using sufficient data in this work.

\section{Methodology}

\subsection{Pre-Processing}

Patient's data were obtained anonymously at the local hospitalwith the specification of the CT Scan: single source detector, 64 slices with the tube voltage of $120 \mathrm{kV}$ producing CT images slices with a thickness of $0.625 \mathrm{~mm}$ and the pixel spacing value $0,488 \mathrm{~mm}$. The input data were consisted of CT scan images from 6 patient's which produced 1639 slices arranged in order according to the slice number from the information of the dicom data. The data was stored in the Graphical User Interface (GUI) "DataStore" for further processing. The preprocessing step was aimed to enhance the edges of CT scan images resulting in an optimal segmentation process. In the early step, the region of interest was determined on a region around the heart and the window level was adjusted to enhance the edges and minimalize parts of the heart that wasn't needed (i.e. fat around the heart).ALaplacian filter was used because it was a derivative filter used to find areas of rapid change (edges) in images. It was particularly good at finding the fine details of an image [20]. As such, this filter type was commonly used in edgedetection applications. Since derivative filters were very sensitive to noise, it was common to smooth the image (using a Gaussian filter) before applying the Laplacian[21, 22]. The Laplacian was a well-known linear differential operator approximating the second derivative by Eq. 1 .

$$
\nabla^{2} f=\frac{\partial^{2} f}{\partial x^{2}}+\frac{\partial^{2} f}{\partial y^{2}}
$$

Where $f$ denotes the image.For convenience computational purposes, Matlab had already provided syntax imgaussfiltandlocallapfitastools for filtering. The results of the pre-processing step was shown in Fig. 1.
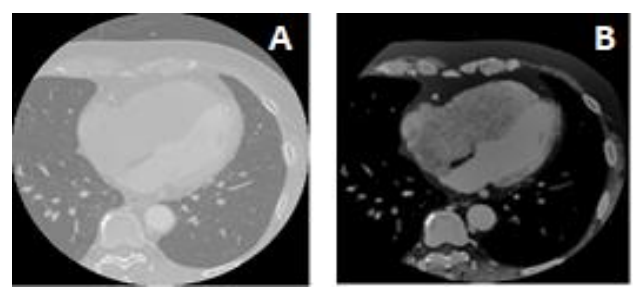

Fig .1: a.Raw data from contrast CT image, b. Image from preprocessing process in matlab.

\subsection{Images Segmentation}

The first step of the segmentation process was to adjust the threshold level to 0.089 using Otsu threshold resulting in a binary image with the matrix range of 0 and 1 . If the CT image was considered as a matrix then the extraction process was conducted by multiplying the matrix of the binary image with the pre-processing image and the result was the extraction image. Afterward, the quality of the extracted image was enhanced using the matlabsyntaxhistaq and was then segmented using active contour algorithm. The input parameterwas given to a region within the extracted image which was referred as masking.As evaluation of the segmentation result, the masking coordinate was adjusted interactively by the user (radiograph) on the central region of the cardiac image for every slice with the same total number of iterations. Parametric active contour are implemented by finding the contour that minimizes energy $E=E_{\text {internal }}+E_{\text {external, }}$ where $E_{\text {internal }}$ was the internal energy of the active contour that quantifies the contour smoothness. The external energy $E_{\text {external }}$ was typically defined such that the contour seeks the edges in images [23].After the masking process, the boundary of the heart was formed, the region was quantified using regionprops in Matlab. This feature result in a value of the segmented area in pixel value which was then converted into $\mathrm{cm}^{2}$ based on the pixel spacing information indicom images.

\subsection{Volume Measurement}

The area of the segmented contour is the area within one slice. For the volume measurement, image process looping is needed from the first slice to $\mathrm{N}$ slice. The $\mathrm{N}$ slice is obtained manually by converting CT image into sagittal and coronal axis to avoid segmentation process of the liver. This step is implemented by converting the image array of every slice, shown in Fig. 3.The slices that contain parts of the liver is removed and only the slices of the heart was used by evaluating the sagittal and coronal images during image processing. The resulted area of each slice is stored and is written in "DataStore" and the volume value of the heart is obtained by implementingthe trapezoidal rule which is the sum of the segmented area of each slice multiplied by the thickness of each slice. 

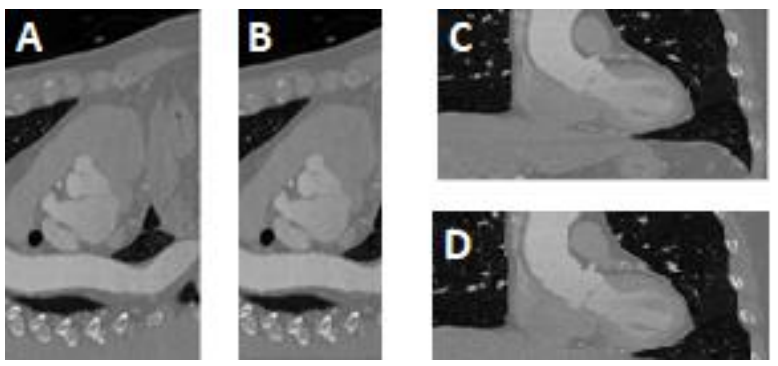

Fig. 3:a. Sagittal image, b. Results of cropping on sagittal image, c. Coronal image, d. Results of cropping on coronal image.

In general, this methodology is explained in Fig. 4. where image processing is a vital process of the quantification of the heart volume.

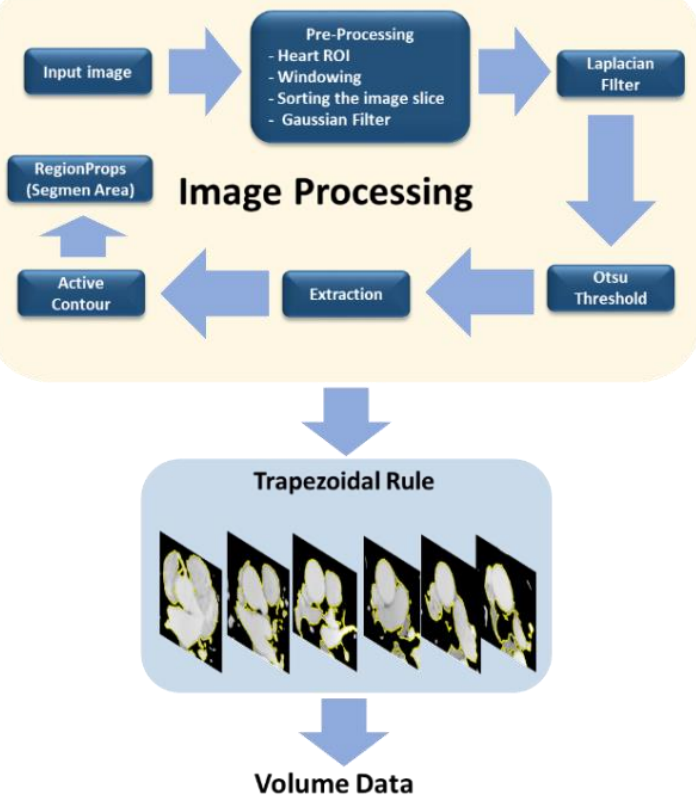

Fig. 4: Flow chart of image processing method and volume quantification.

\section{Results and Discussions}

This study is the first step of the author's research regarding the characterization of heart arteries using volume data of heart which is caused by the minimal volume data information of the heart and the boundaries of the data.Several researches on image processing using the Otsu threshold and active contour segmentation method have been published by $[24,25]$. In those studies, the results of the active contour and threshold segmentation are compared and evaluated using jaccard and dice coefficient. Nevertheless, our own research is more about combining the two models and evaluate the results ot the segmentation, whether if it can properly segment the heart so that our method can be used to quantify the volume of the heart, evidenced by the number of segmentedslicesto make the dataset automatic by visual observation assisted by cardiovascular technicians and radiographers. Evaluation of the segmentation method in this study is conducted by comparing the resulted segmentation of each slice according to the slices of the heart region that are successfully segmented, the heart region with the spinal cord included, other organs, and slices with contour that didn't fully include all the region of the heart.
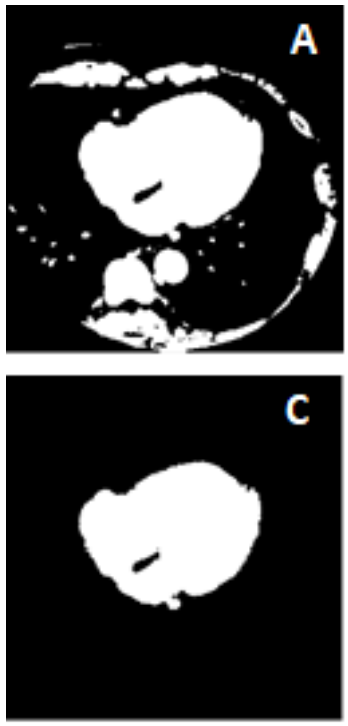

B

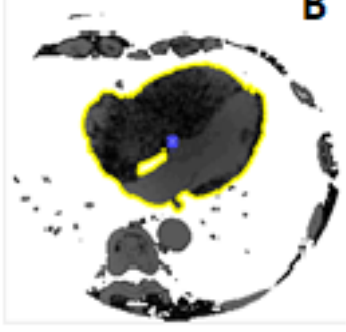

D

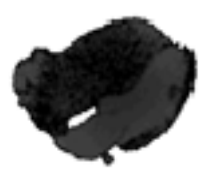

Fig. 5: Image processing results, a. Otsu threshold, b. active contour implementation, c. binary image results from active contour segmentation, d. image extraction.

According to the methodology, the first slice processed is selected when the first coronary artery from the aorta is visible and the last slice is constrained anatomically as the bottom part of the heart, shown in Fig. 3b and Fig. 3d, which resulted in a total of 689 slices chosen from 1639 slices. As part of the analysis method used, the difference of the images during each step of the image processing is shown. During the segmentation step, Otsu threshold algorithm is used to separate the fat surrounding the heart but other objects outside the heart region such as the abdomen, spinal cord, and rib can be seen. To separate those unwanted objects from the Region of Interest (ROI), active contour is implemented in the center area of the heart manually with the supervision from a cardiovascular technician shown as a blue point in Fig. 5b. The resulted image with a boundary of the heart produced a binary image of the heart region. For the next data in this study, the segmented binary image is extracted as an input data for heart volume reconstruction so that the heart can be analyzed in 3D. Not every CT cardiac image covered the heart region, a few segmented image resulted in segmented region outside the region of interest shown in Fig. 6. Anatomically, the heart has four chamber in which functions as heart pumps: right atrium (RA),left atrium (LA), left ventricle (LV), right ventricle (RV).Complications during image segmentation are conventionally due to LA, where anatomically the LA region is the closest to the spinal cord [6], in addition, that part also has the same pixel range as the spinal cord. 


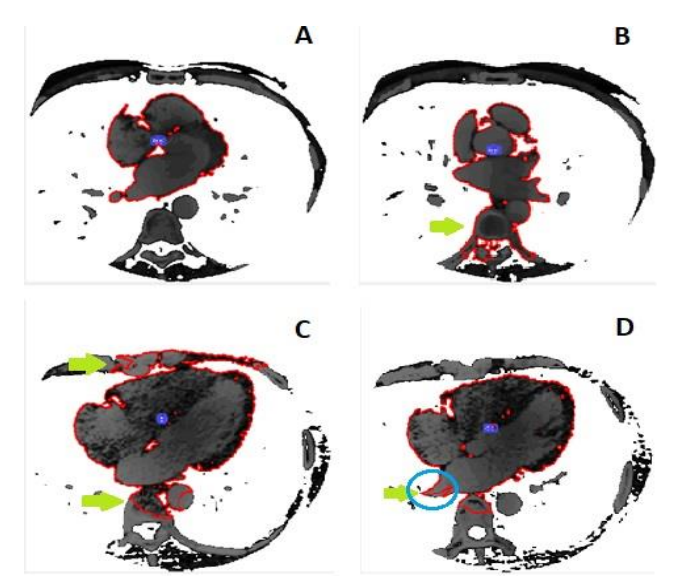

Fig. 6: The contour form that is used as an evaluation, a. contours of the heart area, b. contours that spread to the spine, c. contours that spread to other organs, d. uncovered areas of the heart (highlighted in blue point).
The amount of slices contoured until the spinal cord and other organs are depicted in Table. 1.Window level adjustment is conductedas optimal as possible so that the active contour algorithm can covers the heart region. There are many ways to solve this issue such as adjusting the number of iterations of the active contour. However, this caused regions of the heart that weren't covered in the contourbecauseto detect object in the images, active contour algorithm use the function which stops the level set function evolution at the object boundaries. It's obvious that the evolution is based on the stopping function which depends on the image gradient [24]. Therefore this mode leads to unsatisfactory results in presence of conclusion, low contrast, low iteration, and even noise.

Table 1. Comparison of the results of slice segmentation for each patient.

\begin{tabular}{ccccccc}
\hline \multirow{2}{*}{ Patient } & Slice Used & Body Thickness & \multicolumn{5}{c}{ Segmented Area } \\
\cline { 4 - 6 } & (Slices) & 208 & $\begin{array}{c}\text { Heart } \\
\text { (Slices) }\end{array}$ & $\begin{array}{c}\text { Spine } \\
\text { (Slices) }\end{array}$ & $\begin{array}{c}\text { Other Organs } \\
\text { (Slices) }\end{array}$ & $\begin{array}{c}\text { Uncover } \\
\text { (Slices) }\end{array}$ \\
\hline 1 & 126 & 108 & 6 & 14 & 2 \\
2 & 107 & 205 & 55 & 40 & 47 & 3 \\
3 & 112 & 182 & 61 & 0 & 13 & 1 \\
4 & 132 & 198 & 85 & 11 & 32 & 0 \\
5 & 99 & 205 & 97 & 14 & 4 & 2 \\
6 & 113 & & & & 45 & 1 \\
\hline
\end{tabular}

From the image processing conducted in this study, there were segmented areas(heart, spine, other organs), other organs outside the heart region(abdomenand rib)and spinal cord. The thickness of the body measurement used is obtained by using Image software using the same slice and area. Uncover slicesshows the slice with the contour that didn't include parts of the heart. Based on the data, it is shown that changes of the geometry of thebody's thickness can influence the segmented area such as the patients with a thinner thickness (patient 2 and 4). This can occur when evaluating the morphology of the patient with a thinner thickness, the distance between the heart and the organ or object will be closer but the pixel value range will be the same as that of other thickness. Therefore, when thresholding the segmented image, it is shown that the organ is connected with the region of interest which couldn't be covered properly by the active contour algorithm.This will certainly influence the quantification of the heart volume.This project is focused on making a dataset which can be used as a comparison or ground truth in our research. The information regarding the evaluation during this study will give a further development towards future studies.

Due to the segmentation method being conducted manually, evaluating our measurement results against user dependence is done by the quantitative evaluation of inter-observer variability to produce a linearity of the measurements made by different observers with the patient data taken randomly, in this case we used patient number 5to produce an area size of $\mathrm{cm}^{2}$ every slice shown in Fig. 7.

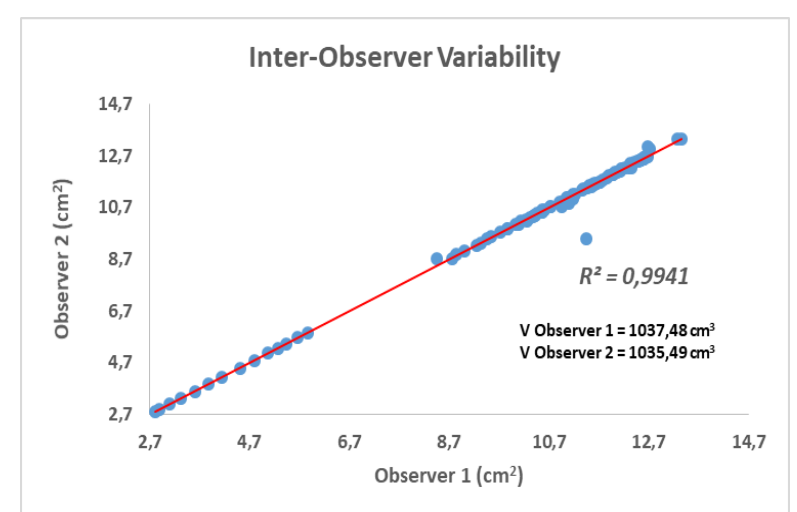

Fig. 7: Quantitative evaluation from two observers using inter-observer variability method.

Based on the evaluation result conducted by two observers: author and a medical physicist, it is shown that there is a correlation between the two measurements shown with the $r$ value of 0,9941 . It is 
expected that the evaluation can be used as data consistency and for the program being used.

\section{Conclusion}

Considering the complexity regarding the morphology of the heart, the proposed segmentation method based on active contour combined with Otsu threshold resulted in an analysis of the segmented region of every slice. From the total 689 slices evaluated, as many as $73.75 \pm 19.41 \%$ of the CT slices are segmented properly, $19.15 \pm 19.61 \%$ of the slices that were segmented included the spine bone, $1.36 \pm 0.98 \%$ of the slices did not include all regions of the heart, $16.58 \pm 15.26 \%$ of the slices.As a quantitative evaluation of the segmentation methods used for volume quantification, the author used inter-observer variability to prove the measurement consistency of the segmentation method done producing $r=0,9941$. However, for the image slice value that didn't cover all the regions of the heart, the value is in agreement with these studies $[14,16,20]$.

\section{Conflict of Interest}

The authors declare that they have no conflict of interest.

\section{References}

[1] M. J. Budoff et al., "Computed Tomography A Scientific Statement From the American Heart Association Committee on Cardiovascular Imaging and Intervention, Council on Cardiovascular Radiology and Intervention, and Committee on Cardiac Imaging," Counc. Clinic. Cardiol.,(2006).

[2] M. A. Hussain, A. Al Mamun, S. A. E. Peters, M. Woodward, and R. R. Huxley, "The Burden of Cardiovascular Disease Attributable to Major Modi fi able Risk Factors in Indonesia," J. Epidemiol., 26, 10, 515-521, (2016).

[3] J. Leipsic et al., "ScienceDirect SCCT Guidelines SCCT guidelines for the interpretation and reporting of coronary CT angiography : A report of the Society of Cardiovascular Computed Tomography Guidelines Committee," J. Cardiovasc. Comput. Tomogr., 8,5, 342-358, (2014).

[4] M. A. De Graaf et al., "Feasibility of an Automated Quantitative Computed Tomography Angiography e Derived Risk Score for Risk Strati fi cation of Patients With Suspected Coronary Artery Disease," Am. J. Cardiol., 5, 4, 1-9, (2014).

[5] G. Yang and Y. Chen, "Automatic coronary calcium scoring using noncontrast and contrast CT images," Med. Phys., 43, 2, 2174-2186, (2016).

[6] P. J. Bazira, "Clinically applied anatomy of the heart," Surgery, 39, 3, 117-125, (2021).

[7] W. T Van. Shahzad, Rahiil, D. Bos, R. P. Budde, K. Pellikaan, W. J. Niessen, and V. D. Lugt, "Automatic segmentation and quantification of the cardiac structures from non-contrast-enhanced cardiac CT scans," Phys. Med. Biology, 3798-3813, (2017).

[8] J. Larrey-ruiz, J. Morales-sánchez, M. C. Bastidajumilla, R. M. Menchón-lara, R. Verdú-monedero, and J. L. Sancho-gómez, "Automatic imagebased segmentation of the heart from CT scans," J. Image Video Process., 1-13, (2014).

[9] M. Habijan, H. Leventic, I. Galic, and D. Babin, "Whole Heart Segmentation from CT images Using 3D U-Net architecture," 121-126, (2019).

[10] N. Shameena and R. Jabbar, "A study of preprocessing and segmentation techniques on cardiac medical images," IJERT, 3, 4, 336-341, (2014).

[11] D. Cremers, "A Review of Statistical Approaches to Level Set Segmentation: Integrating Color, Texture , Motion and Shape," Int. J. Comput. Vis., 72, 2, 195-215, (2007).

[12] J. Freixenet, X. Cuf, and J. Mart, "Strategies for image segmentation combining region and boundary information," Pattern Recognit. Lett., 24, 375-392, (2003).

[13] T. Y. Goh, S. N. Basah, H. Yazid, M. Juhairi, and A. Safar, "Performance analysis of image thresholding: Otsu technique," Measurement, 114, 298-307, (2018).

[14] C. Li, C. Xu, S. Member, C. Gui, and M. D. Fox, "Distance Regularized Level Set Evolution and Its Application to Image Segmentation," (2010).

[15] Y. Song, G. Peng, D. Sun, and X. Xie, "Active contours driven by Gaussian function and adaptive-scale local correntropy-based Kmeans clustering for fast image segmentation," Signal Processing, 174, (2020).

[16] D. Terzopoulost, J. Platt, and K. Fleischert, "Elastically Deformable Models," Int. J. Comput. Vis., 21, 4, 205-214, (1987).

[17] M. Jolly, "Automatic Segmentation of the Left Ventricle in Cardiac MR and CT Images," Int. J. Comput. Vis., 70, 2, 151-163, (2006).

[18] Y. Lu, P. Radau, K. Connelly, A. Dick, and G. A. Wright, "Segmentation of Left Ventricle in Cardiac Cine MRI : An Automatic Image-Driven Method," 339-340, (2009).

[19] M. Hajiaghayi, D. Ph, E. M. Groves, M. Eng, H. Jafarkhani, and D. Ph, "Transactions on Biomedical Engineering A 3D Active Contour Method for Automated Segmentation of the Left Ventricle from Magnetic Resonance Images," in IEEE Transaction of Biomed. Eng., 9294, (2016).

[20] S. S. Bhairannawar, "Efficient Medical Image Enhancement Technique Using Transform HSV Space and Adaptive Histogram Equalization,"Elsevier Inc., (2018).

[21] R. Hummel, "Image Enhancement by Histogram Transformation*," Comput. Graph. Image Process., 6, 2, 184-195, (1977).

[22] M. Huang, Z. Mu, H. Zeng, and H. Huang, "A Novel Approach for Interest Point Detection via Laplacian-of-Bilateral Filter," J. Sensors, 2015, (2015). 
[23] D. Geoff, Digital image processing for medical applications. USA: Cambridge University Press, (2011).

[24] S. Husham et al., "Comparative Analysis between Active Contour and Otsu Thresholding Segmentation Algorithms in Segmenting Brain Tumor Magnetic Resonance Imaging," J. Inf. Technol. Manag., vol. 12, no. Special Issue: Deep
Learning for Visual Information Analytics and Management., 48-61, (2020).

[25] V. Rajinikanth, N. Madhavaraja, S. Satapathy, and S. Fernandes, "Otsu's Multi-Thresholding and Active Contour Snake Model to Segment Dermoscopy Images," J. Med. Imaging Heal. Informatics, 7, 1837-1840, (2017). 